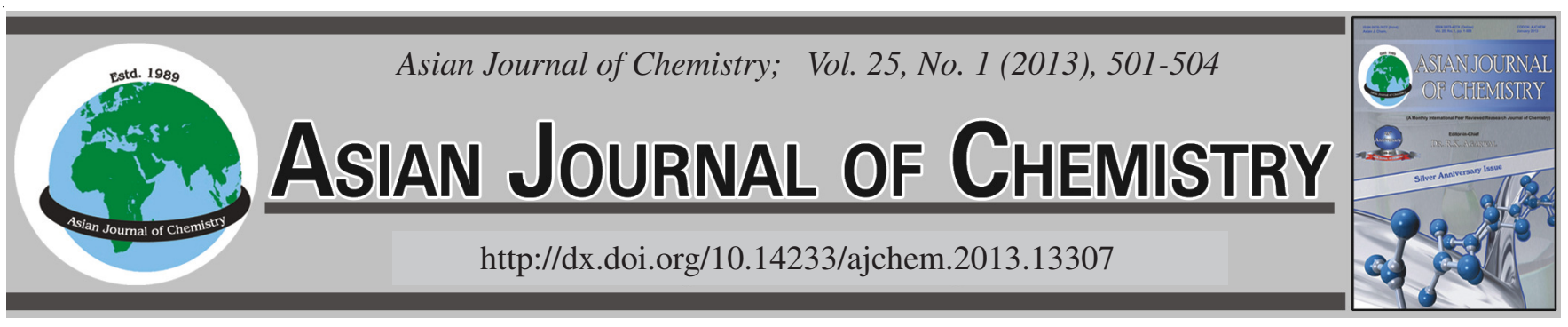

\title{
Synthesis of Heteroaromatic Derivatives with Nitrogen Atoms: Tripyrrolyl Pyrimidine and Tripyrrolyl[1,3,5]triazine
}

\author{
D.H. LEE ${ }^{1}$, S.G. LEE ${ }^{1}$, D.I. JUNG ${ }^{1, *}$ and J.T. HAHN ${ }^{2}$
}

${ }^{1}$ Department of Chemistry, Dong-A University, Busan 604714, South Korea

${ }^{2}$ Department of Beautycare, Youngdong University, Youngdong 370701, South Korea

*Corresponding author: Fax: +82 51 2007249; Tel: +82 51 2007249; E-mail : dijung@dau.ac.kr

As a part of a research program related to the synthetic study of pharmacologically and photoconductively interesting pyrrole derivatives, we have synthesized 1-arylpyrroles (3a-e), 9-arylcarbazoles (4a-e), aminophenylpyrroles (6a,b), dipyrrolylbenzenes (7a-c), 2,4,6-tri-pyrrol-1yl-pyrimidine (8) and 2,4,6-tri-pyrrol-1-yl[1,3,5]triazine (9). We proposed a plausible mechanism for the formation of 9-arylcarbazole.

Key Words: Arylpyrroles, Arylcarbazoles, Diphenylbenzenes, Tripyrrolylpyrimidine, Tripyrrolyl[1,3,5]triazine.

\section{INTRODUCTION}

Nitrogen containing heterocycles, such as pyrroles and carbazoles have attracted considerable attention due to their numerous applications in pharmaceutical and synthetic chemistry ${ }^{1}$. These heterocyclic moieties are also found in a variety of biologically active synthetic and natural products ${ }^{2}$. Although many efficient processes had already been reported, but, the development of new methods is still in demand ${ }^{3}$. Most methods involve two or more steps to synthesize these heterocycles resulting in 2,3-di- or polysubstituted products ${ }^{4}$. Ideally the synthesis of these heterocycles would involve only one step, directly from simple, readily available substrates. Although, a similar idea had been proposed earlier, it suffered serious drawbacks such as low yields (up to $50 \%$ ) and low selectivities $^{4,5}$. In present study, we report a convenient one pot synthesis of $N$-pyrrolylbenzenes and carbazoles from commercially available aromatic amines using glacial acetic acid as an effective catalyst. In the course of the investigation for the synthesis of pyrrole derivatives (3), we have found the formation of 9-arylcarbazoles (4) under refluxing glacial acetic acid. Thus we report the results here. 1-Arylpyrroles were prepared by the previously published procedure ${ }^{5}$.

\section{EXPERIMENTAL}

Melting points were determines on a Büchi 510 capillary melting point apparatus and uncorrected. Infrared spectra were recorded on a Perkin-Elmer 683 spectro-photometer. NMR spectra were recorded on a Varian XL-300 or Brüker AC 200 FT-NMR spectrometer in $\mathrm{CDCl}_{3}$ containing $\mathrm{Me}_{4} \mathrm{Si}$ as an internal reference. Mass spectra were obtained by using JEOL JMS DX 303 or HP 5892 Mass Spectrometer.

Preparation of 9-phenylcarbazole (4c) in glacial acetic acid: A mixture of $\mathbf{3 c}(0.72 \mathrm{~g}, 5 \mathrm{mmol})$ and $\mathbf{2}(1.32 \mathrm{~g}, 10 \mathrm{mmol})$ in glacial acetic acid was refluxed for $19 \mathrm{~h}$. Removal of the solvent under reduced but pressure followed by flash column chromatography on a silica-gel ( $n$-hexane:ethyl acetate $=10: 1$, $\mathrm{v} / \mathrm{v})$ gave the desired 9-phenylcarbazole $4 \mathbf{c}$ as a solid $(0.67 \mathrm{~g}$, $55 \%$ ); m.p. $94-96^{\circ} \mathrm{C}$; IR (KBr, $\left.v_{\max }, \mathrm{cm}^{-1}\right) 3050$ (aromatic C-H) 1590, 1240, 760; ${ }^{1} \mathrm{H} \mathrm{NMR}\left(\mathrm{CDCl}_{3}, 200 \mathrm{MHz}\right) \delta$ 7.25-8.18 (m, $13 \mathrm{H}$, phenyl or carbazolyl group); ${ }^{13} \mathrm{C} \mathrm{NMR}\left(\mathrm{CDCl}_{3}, 50.32\right.$ MHz) $\delta 129.9,127.5,125.9,120.3,119.9,109.8$; Mass (m/e) $243\left(\mathrm{M}^{+}\right), 166,140,77$.

Preparation of 9-(4'-methoxyphenyl)carbazole (4a) by direct and one-pot reaction in glacial acetic acid: A mixture of $1 \mathbf{a}(1.85 \mathrm{~g}, 15 \mathrm{mmol})$ and $2(6.20 \mathrm{~g}, 45 \mathrm{mmol})$ in glacial acetic acid was refluxed for $12 \mathrm{~h}$. The solvent was removed under aspirator pressure and the remaining sticky oil was separated by flash column chromatography on a silica gel ( $n$-hexane). Yield $1.23 \mathrm{~g}(30 \%)$; IR $\left(\mathrm{KBr}, \mathrm{v}_{\max }, \mathrm{cm}^{-1}\right) 3070$ (aromatic C-H) 2950, 1600, 1210, 1120, 800; ${ }^{1} \mathrm{H}$ NMR $\left(\mathrm{CDCl}_{3}, 200 \mathrm{MHz}\right) \delta$ $3.66\left(\mathrm{~s}, 3 \mathrm{H}, \mathrm{CH}_{3}\right) 7.25-8.18(\mathrm{~m}, 12 \mathrm{H}$, phenyl and carbazolyl group); Mass (m/e) $273\left(\mathrm{M}^{+}\right), 258,242,166$.

Physical data of 1-arylpyrroles: $1{ }^{1} \mathrm{H}$ NMR $\left(\mathrm{CDCl}_{3}\right.$, $200 \mathrm{MHz}) \delta 3.83\left(\mathrm{~s}, 3 \mathrm{H}, \mathrm{CH}_{3}\right), 6.32(\mathrm{t}, 2 \mathrm{H}), 6.92-6.99(\mathrm{~m}$, $2 \mathrm{H}), 7.28-7.33(\mathrm{~m}, 2 \mathrm{H})$; Mass $(\mathrm{m} / \mathrm{e}) 173\left(\mathrm{M}^{+}\right)$. 1b ${ }^{1} \mathrm{H}$ NMR $\left(\mathrm{CDCl}_{3}, 200 \mathrm{MHz}\right) \delta 6.41-6.44(\mathrm{t}, 2 \mathrm{H}), 7.16-7.18(\mathrm{t}, 2 \mathrm{H}), 7.49-$ 7.54 (m, 2H), 8.28-8.33 (m, 2H). 1c ${ }^{1} \mathrm{H} \mathrm{NMR}\left(\mathrm{CDCl}_{3}, 200\right.$ $\mathrm{MHz}) \delta$ 6.33-6.35 (t, 2H), 7.08-7.10 (t, 2H), 7.23-7.24 (m, 1H), 7.39-7.42 (m, 4H); Mass (m/e) $143\left(\mathrm{M}^{+}\right)$. 1d Mass (m/e) 
$188\left(\mathrm{M}^{+}\right) .1 \mathrm{e}$ Mass $(\mathrm{m} / \mathrm{e}) 222\left(\mathrm{M}^{+}\right)$.

Physical data of 9-arylcarbazoles: $4 \mathrm{a} I \mathrm{IR}\left(\mathrm{KBr}, \mathrm{v}_{\max }, \mathrm{cm}^{-1}\right)$ 3070-2980 w (aromatic C-H), 1600-1400s (aromatic C=C), $1120 \mathrm{~s}(\mathrm{C}-\mathrm{O}), 1210 \mathrm{~s}(\mathrm{C}-\mathrm{N}), 800-650 \mathrm{w}$ (=CH, aromatic OOP); ${ }^{1} \mathrm{H}$ NMR $\left(\mathrm{CDCl}_{3}, 200 \mathrm{MHz}\right) \delta 3.83\left(\mathrm{~s}, 3 \mathrm{H}, \mathrm{CH}_{3}\right), 7.02-8.19$ (m, 12H, phenyl and carbazolyl group); Mass (m/e): 274 $\left(\mathrm{M}^{+}+1,25\right), 273\left(\mathrm{M}^{+}, 100\right), 258,242,166.4 b$ IR (neat) 30502900w (aromatic C-H), 1550s, 1390s $\left(\mathrm{NO}_{2}\right), 1500-1450$ s (aromatic $\mathrm{C}=\mathrm{C}), 1230 \mathrm{~s}(\mathrm{C}-\mathrm{N}), 760-720 \mathrm{w}(=\mathrm{CH}$. Aromatic OOP $) \mathrm{cm}^{-1} ;{ }^{1} \mathrm{H} \mathrm{NMR}\left(\mathrm{CDCl}_{3}, 300 \mathrm{MHz}\right) \delta 6.34-7.68(\mathrm{~m}, 12 \mathrm{H}$, phenyl and carbazolyl group); Mass (m/e) $268\left(\mathrm{M}^{+}\right), 242,166$, 140, 46, 30. 4c IR (KBr, $\left.v_{\max }, \mathrm{cm}^{-1}\right)$ 3050-2950w (aromatic C-H), 1590s (aromatic C=C), 1240s $(\mathrm{C}-\mathrm{N}), 760-700$ s $(=\mathrm{CH}$ aromatic OOP); ${ }^{1} \mathrm{H} \mathrm{NMR}\left(\mathrm{CDCl}_{3}, 200 \mathrm{MHz}\right) \delta 7.20-8.18(\mathrm{~m}$, $13 \mathrm{H}$, phenyl and carbazolyl group); ${ }^{13} \mathrm{C} \mathrm{NMR}\left(\mathrm{CDCl}_{3}, 50.32\right.$ MHz) $\delta 129.89,127.45,125.91,120.30,119.89,109.76$; Mass (m/e): $244\left(\mathrm{M}^{+}+1,23\right), 243\left(\mathrm{M}^{+}, 100\right), 166,140,77.4 d \mathrm{IR}$ (neat) 3050-2900w (aromatic C-H), 1550s, 1390s $\left(\mathrm{NO}_{2}\right), 1500$ $1450 \mathrm{~s}$ (aromatic $\mathrm{C}=\mathrm{C}), 1230 \mathrm{~s}(\mathrm{C}-\mathrm{N}), 760-720 \mathrm{w}(=\mathrm{CH}$, aromatic OOP) $\mathrm{cm}^{-1}$; ${ }^{1} \mathrm{H} \mathrm{NMR}\left(\mathrm{CDCl}_{3}, 300 \mathrm{MHz}\right) \delta 6.34-7.68$ (m, phenyl and carbazolyl group); Mass (m/e): 288, 242, 166, 140, 46, 30. 4e IR (neat) 3030-3010w (aromatic C-H), 1500-1450s (aromatic $\mathrm{C}=\mathrm{C}), 1215 \mathrm{~s}(\mathrm{C}-\mathrm{N}), 1005 \mathrm{~s}(\operatorname{aryl}-\mathrm{Br}), 770-710 \mathrm{~s}$ $(=\mathrm{CH}$, aromatic OOP $) ;{ }^{1} \mathrm{H} \mathrm{NMR}\left(\mathrm{CDCl}_{3}, 200 \mathrm{MHz}\right) \delta 7.25-$ 8.20 (m, 12H, phenyl and carbazolyl group); Mass (m/e): 323 $\left(\mathrm{M}^{+}+2,103\right), 321\left(\mathrm{M}^{+}, 100\right), 241,166,140,76$.

1-(2-aminophe-nyl)pyrrole (6a): Yield : $73 \%$; m.p.: 102$103^{\circ} \mathrm{C} ; \mathrm{R}_{\mathrm{f}}: 0.44$ (TLC eluent; $n$-hexane : EtOAc $=20: 1, \mathrm{v} / \mathrm{v}$ ); ${ }^{1} \mathrm{H}$ NMR $\left(\mathrm{CDCl}_{3}, 200 \mathrm{MHz}\right): \delta 3.68(\mathrm{~s}, 2 \mathrm{H}), 6.31-6.35$, (s, 2H), 6.72-6.78 (m, 2H), 6.79-6.82 (s, 4H), 7.10-7.15 (m, 2H); Mass (70eV), m/z : 158.

1,2-Dipyrrolylbenzene (7a): Isolated yield : $5 \%$; $\mathrm{R}_{\mathrm{f}}$ : 0.68 (TLC eluent ; EtOAc : $n$-hexane $=1: 20, \mathrm{v} / \mathrm{v})$; Mass $(70 \mathrm{eV})$, m/z (rel. Int. \%) : 208 (19), 158 (62), 132 (71), 77 (71), 63 (90), 51 (100); ${ }^{1} \mathrm{H}$ NMR $\left(\mathrm{CDCl}_{3}, 200 \mathrm{MHz}\right): \delta 7.62(\mathrm{~m}, 2 \mathrm{H})$, $\delta 7.58(\mathrm{~m}, 2 \mathrm{H}), \delta 7.26(\mathrm{~m}, 4 \mathrm{H}) \delta 6.41(\mathrm{~m}, 4 \mathrm{H}) ;{ }^{13} \mathrm{C} \mathrm{NMR}$ $\left(\mathrm{CDCl}_{3}, 50 \mathrm{MHz}\right): \delta 143.7,131.3,131.0,130.6,129.4,122.6$, 120.4, 120.1, 110.5, 108.9, 48.9, 29.6; Anal. calcd. for $\mathrm{C}_{14} \mathrm{H}_{12} \mathrm{~N}_{2}: \mathrm{C}, 80.74 ; \mathrm{H}, 5.81 ; \mathrm{N}, 13.45$; Found : C, 80.71; H, $5.80 ; \mathrm{N}, 13.43$.

1-(3-Aminophenyl)-pyrrole (6b): Yield: $28 \%$; m.p.: 120$122{ }^{\circ} \mathrm{C} ; \mathrm{R}_{\mathrm{f}}: 0.44$ (TLC eluent; $n$-hexane : EtOAc $=20: 1, \mathrm{v} / \mathrm{v}$ ); ${ }^{1} \mathrm{H} \mathrm{NMR}\left(\mathrm{CDCl}_{3}, 200 \mathrm{MHz}\right): \delta 2.20(\mathrm{~s}, 2 \mathrm{H}), 6.20(\mathrm{~s}, 2 \mathrm{H}), 7.10$ (m, 2H), 7.30 (s, 2H); Mass (70 eV), m/z: 158.

1,3-Dipyrrolylbenzene (7b): Yield: 37 \%; m.p.: 107$108{ }^{\circ} \mathrm{C} ; \mathrm{R}_{\mathrm{f}}: 0.675$ (TLC eluent; $n$-hexane: EtOAc = $5: 1, \mathrm{v} / \mathrm{v}$ ); ${ }^{1} \mathrm{H} \mathrm{NMR}\left(\mathrm{CDCl}_{3}, 200 \mathrm{MHz}\right): \delta 6.36-6.38(\mathrm{t}, 4 \mathrm{H}), 7.10-7.12(\mathrm{t}$, 4H), 7.25-7.29 (m, 3H), 7.41-7.60 (m, 1H) Mass (70eV), m/z: 208.

1,4-Dipyrrolyl-benzene (7c): Yield : $58 \%$; m.p. : 213 $214{ }^{\circ} \mathrm{C} ; \mathrm{R}_{\mathrm{f}}: 0.44$ (TLC eluent; $n$-hexane: EtOAc $=10: 1$, $\mathrm{v} / \mathrm{v}) ;{ }^{1} \mathrm{H}$ NMR $\left(\mathrm{CDCl}_{3}, 200 \mathrm{MHz}\right): \delta 6.17-6.22(\mathrm{t}, 4 \mathrm{H}), 6.89$ $6.96(\mathrm{~m}, 4 \mathrm{H}), 7.16(\mathrm{~m}, 2 \mathrm{H}), 7.36(\mathrm{~m}, 2 \mathrm{H})$; Mass $(70 \mathrm{eV}), \mathrm{m} / \mathrm{z}$ : 208.

2,4,6-Tripyrrol-1-yl-pyrimidine (8): Isolated yield: $30 \%$ (39.5\%); m.p. : $171-173{ }^{\circ} \mathrm{C} ; \mathrm{R}_{\mathrm{f}}: 0.26$ (TLC eluent; EtOAc: $n$-hexane $=1: 40, \mathrm{v} / \mathrm{v}) ;$ Mass $(70 \mathrm{eV}), \mathrm{m} / \mathrm{z}$ (rel. Int. \%): 275 (10), 51 (100), 129 (30), 75 (30), 102 (20); ${ }^{1} \mathrm{H} \mathrm{NMR}\left(\mathrm{CDCl}_{3}\right.$, $200 \mathrm{MHz}): \delta 7.82-7.84(\mathrm{t}, 3 \mathrm{H}), 7.61-7.62(\mathrm{t}, 4 \mathrm{H}), 6.84(\mathrm{~s}, 2 \mathrm{H})$,
6.42-6.44 (t, 3H), 6.35-6.37 (t, 3H); ${ }^{13} \mathrm{C} \mathrm{NMR}\left(\mathrm{CDCl}_{3}, 50\right.$ $\mathrm{MHz}): \delta 159.1,119.51,118.98,118.50,118.03,114.44$, 113.16, 112.74, 112.35, 111.88, 111.51, 88.23; Anal. calcd. for $\mathrm{C}_{16} \mathrm{H}_{13} \mathrm{~N}_{5}$ : C, 69.80; H, 4.76; N, 25.44; Found: C, 69.78; H, 4.74, N, 25.41.

2,4,6-Tri-pyrrol-1-yl-[1,3,5]triazine (9): Isolated yield : $29 \%$ (30\%); m.p.: $187-189^{\circ} \mathrm{C} ; \mathrm{R}_{\mathrm{f}}$ : 0.35 (TLC eluent; $\mathrm{CHCl}_{3}$ : $n$-hexane $=1: 40, \mathrm{v} / \mathrm{v}) ;$ Mass $(70 \mathrm{eV}), \mathrm{m} / \mathrm{z}$ (rel. Int. \%): 276 (10), 51 (100), 129 (30), 75 (30), 102 (20); ${ }^{1} \mathrm{H} \mathrm{NMR} \mathrm{(CDCl}{ }_{3}$, $200 \mathrm{MHz}): \delta$ 7.81-7.84 (t, 6H), 6.39-6.41 (t, 6H); ${ }^{13} \mathrm{C} \mathrm{NMR}$ $\left(\mathrm{CDCl}_{3}, 50 \mathrm{MHz}\right): \delta 182.21,138.88,138.36,132.95,132.41$.

\section{RESULTS AND DISCUSSION}

Generarlly, synthetic methods of 1-arylpyrroles 3 from amines $\mathbf{1}$ and $\mathbf{2}$ have been known for a long time ${ }^{8-11}$. 1-Arylpyrroles 3 were obtained in quantitative yields by the general method (Table-1). The effect of organic dicarboxylic acids on the synthesis of $\mathbf{3}$ was investigated (Table-2). Among organic dicarboxylic acids, adipic acid gave the highest yield of $\mathbf{3 c}$ (Table-2, Entry 1) (Scheme-I).

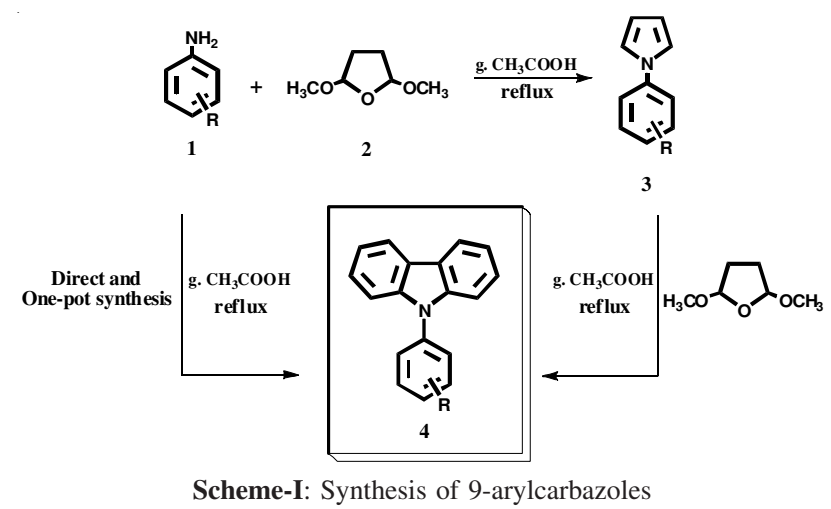

The yield of $\mathbf{3 c}$ was the lowest when acetonedicarboxylic acid was used (Table 2, Entry 3), but $N$-phenylnortropinone was formed as the major product in $48 \%$ yield.

TABLE-1

PHYSICAL DATA OF 1-ARYLPYRROLES (3)

\begin{tabular}{llccc}
\hline \multicolumn{1}{c}{$\mathrm{R}$} & Yield $(\%)^{\mathrm{a}}$ & m.p. $\left({ }^{\circ} \mathrm{C}\right)$ & Lit. ${ }^{8}$ m.p. $\left({ }^{\circ} \mathrm{C}\right)$ \\
\hline a & $p-\mathrm{OCH}_{3}$ & 98 & $108-109$ & 108 \\
b & $p-\mathrm{NO}_{2}$ & 88 & $180-181$ & $180-181$ \\
c & $\mathrm{H}$ & 98 & $58-59^{6,7}$ & $58-59$ \\
d & $m-\mathrm{NO}_{2}$ & 87 & $81-82^{7}$ & $81-82$ \\
e & $m-\mathrm{Br}$ & 93 & $64-65^{6,7}$ & - \\
\hline${ }^{\text {a }}$ Isolated yield & & &
\end{tabular}

TABLE-2

YIELDS OF 1-PHENYLPYRROLE (3c) DEPENDING ON THE DICARBOXYLIC ACIDS

\begin{tabular}{clcc}
\hline Entry & \multicolumn{1}{c}{ Organic dicarboxylic acid } & $\begin{array}{c}\text { Refiux } \\
(\mathrm{min})\end{array}$ & $\begin{array}{c}\text { Yield } \\
(\%)^{\mathrm{a}} \mathbf{3 c}\end{array}$ \\
\hline $\mathbf{1}$ & Adipic acid & 75 & 87 \\
$\mathbf{2}$ & Tartaric acid & 60 & 22 \\
$\mathbf{3}$ & Acetonedicarboxylic acid & 40 & $2(48)^{\mathrm{b}}$ \\
$\mathbf{4}$ & 3-(Carboxymethylthio)propionic acid & 240 & 42 \\
$\mathbf{5}$ & 2-Ketoglutaric acid & 30 & 8 \\
\hline${ }^{\mathrm{a}}$ Isolated yield. ${ }^{\mathrm{b}} N$-Phenylnortropinone & & \\
\end{tabular}

9-Arylcarbazoles (4) were formed by treatment of 1-aryl- 
pyrroles (3) with $\mathbf{2}$ in glacial acetic acid. The yield of 4's were summerized depending on the substituent(R) in Table-3.

\begin{tabular}{llccc}
\hline \multicolumn{5}{c}{ TABLE-3 } \\
\multicolumn{5}{c}{ PHYSICAL DATA OF 9-ARYLCARBAZOLES 4 } \\
\hline & $\mathrm{R}$ & Reflux (h) & Yield $(\%)^{\mathrm{a}}$ & m.p. $\left({ }^{\circ} \mathrm{C}\right)$ \\
\hline a & $p-\mathrm{OCH}_{3}$ & 15 & 54 & $149-150$ \\
b & $p-\mathrm{NO}_{2}$ & 28 & 30 & b \\
c & $\mathrm{H}$ & 19 & 55 & $94-96$ \\
d & $m-\mathrm{NO}_{2}$ & 29 & 24 & $119-121$ \\
e & $m$ - $\mathrm{Br}$ & 18 & 23 & b \\
\hline 'Isolated yield; ${ }^{b}$ Liquid & & &
\end{tabular}

A representative example of synthesis $\mathbf{4}$ is as the fol-low. The mixture of $\mathbf{3 c}(5 \mathrm{mmol})$ and $\mathbf{2}(10 \mathrm{mmol})$ was refluxed in glacial acetic acid under $\mathrm{N}_{2}$ gas for $19 \mathrm{~h}$ to afford $4 \mathrm{c}$ in a $55 \%$ yield. Identification of 9-phenylcarbazole by ${ }^{1} \mathrm{H}$ NMR spectrum $\left(\mathrm{CDCl}_{3}, \mathrm{Me}_{4} \mathrm{Si}\right)$ showed 13 proton peaks correspoding to carbazolyl group and phenyl group at $\delta 7.25-8.18$. Mass spectrum showed molecular ion peaks at m/e $243(100 \%)$.

But the synthesis of 9-alkylcarbazoles from the corresponding 1-alkylpyrroles was not successful. 9-Arylcarbazoles (4) scan also be prepared by one-pot reactions of the aromatic amines $\mathbf{1}$ and $\mathbf{2}$ in glacial acetic acid under $\mathrm{N}_{2}$ gas. The results are listed in Table-4. However, the yields from the one-pot reaction are much lower than the reaction from 1-arylpyrroles. In order to synthesize dipyrrolylbenzenes $\mathbf{7 a - c}$ are, reactions of phenylenediamines 5a-c and 2,5-dimethoxytetrahydrofuran with glacial acetic acid as a catalyst were executed.

TABLE-4

ONE-POT SYNTHESIS OF 9-ARYL CARBAZOLES 4

\begin{tabular}{clcc}
\hline & $\mathrm{R}$ & Reflux $(\mathrm{h})$ & ${\text { Yield }(\%)^{\mathrm{a}}}^{\mathrm{a}}$ \\
\hline $\mathbf{a}$ & $p-\mathrm{OCH}_{3}$ & 12 & 30 \\
$\mathbf{b}$ & $p-\mathrm{NO}_{2}$ & 20 & 23 \\
c & $\mathrm{H}$ & 10 & 47 \\
d & $m-\mathrm{NO}_{2}$ & 32 & 20 \\
e & $m-\mathrm{Br}$ & 18 & 15 \\
\hline asolated yield & &
\end{tabular}

a. ortho-

b. meta-

c. para-

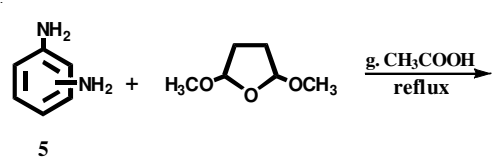

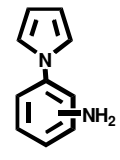

6 a. orthob. meta-

c. para-
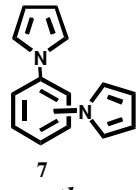

a. ortho-

b. meta-

c. para-
Scheme-II: Aminophenylpyrroles and dipyrrolylbenzenes

The synthetic results of aminophenylpyrroles $\mathbf{6 a - c}$ and dipyrrolylbenzenes $\mathbf{7 a - c}$ are listed in Table-5.

In case of $\mathbf{5 c}, \mathbf{7 c}$ as a dipyrrolylbenzene was obtained in a $58 \%$ yield but $\mathbf{6 c}$ as a aminophenylpyrrole was not obtained. 2,4,6-Tri-pyrrol-1-yl-pyrimidine (8) bearing tripyrrolyl groups was formed by treatment of 2,4,6-triaminopyrimidine and 2,5dimethoxytetrahydrofuran with glacial acetic acid. Yield of 8 obtained in reflux for $13 \mathrm{~h}$ is $37 \%$ and yield of $\mathbf{8}$ obtained in sealed tube for $0.5 \mathrm{~h}$ is $39.5 \%$. In same synthetic method, 2,4,6-tri-pyrrol-1-yl[1,3,5]triazine (9) bearing tripyrrolyl groups was formed in a $29 \%$ yield (in reflux for $13 \mathrm{~h}$ ) and in a $30 \%$ yield (in sealed tube) (Scheme-III).

\begin{tabular}{|c|c|c|c|c|c|}
\hline \multicolumn{6}{|c|}{$\begin{array}{c}\text { TABLE-5 } \\
\text { PHYSICAL DATA OF AMINOPHENYLPYRROLES } \\
\text { 6a-c AND DIPYRROLYLBENZENES 7a-c }\end{array}$} \\
\hline Reactant & Solvent & $\begin{array}{l}\text { Reflux } \\
\text { (h) }\end{array}$ & $\begin{array}{c}\text { Product } \\
\text { No. }\end{array}$ & $\begin{array}{l}\text { Yield } \\
(\%)^{*}\end{array}$ & $\begin{array}{l}\text { m.p. } \\
\left({ }^{\circ} \mathrm{C}\right)\end{array}$ \\
\hline \multirow{2}{*}{$5 a$} & \multirow{2}{*}{ g.AcOH } & \multirow{2}{*}{6} & $6 a$ & 73 & $102-103$ \\
\hline & & & $7 a$ & 5 & 78-79 \\
\hline \multirow{2}{*}{$5 b$} & & \multirow[b]{2}{*}{1} & $6 b$ & 28 & $120-122$ \\
\hline & & & $7 \mathrm{~b}$ & 37 & $107-108$ \\
\hline \multirow{2}{*}{$5 c$} & & \multirow[b]{2}{*}{1} & $6 c$ & - & - \\
\hline & & & $7 \mathrm{c}$ & 58 & $213-214$ \\
\hline
\end{tabular}

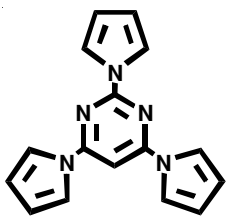

8

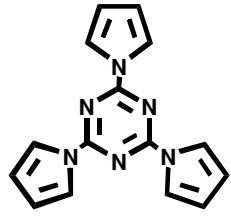

Scheme-III: 2,4,6-Tri-pyrrol-1-yl-pyrimidine (8) and 2,4,6-tri-pyrrol-1yl[1,3,5]triazine $(9)$

In order to investigate the mechanism, the products in the reaction mixture were monitored with time by gas chromatography. 1-Arylindoles were detec-ted by gas chromatography, which were comfirmed with the authentic samples. A possible mechanism for the formation of $\mathbf{4}$ may involve the cleavage reaction of furan ring by glacial acetic acid and subsequent formation of intermediates X and Y (Scheme-IV).

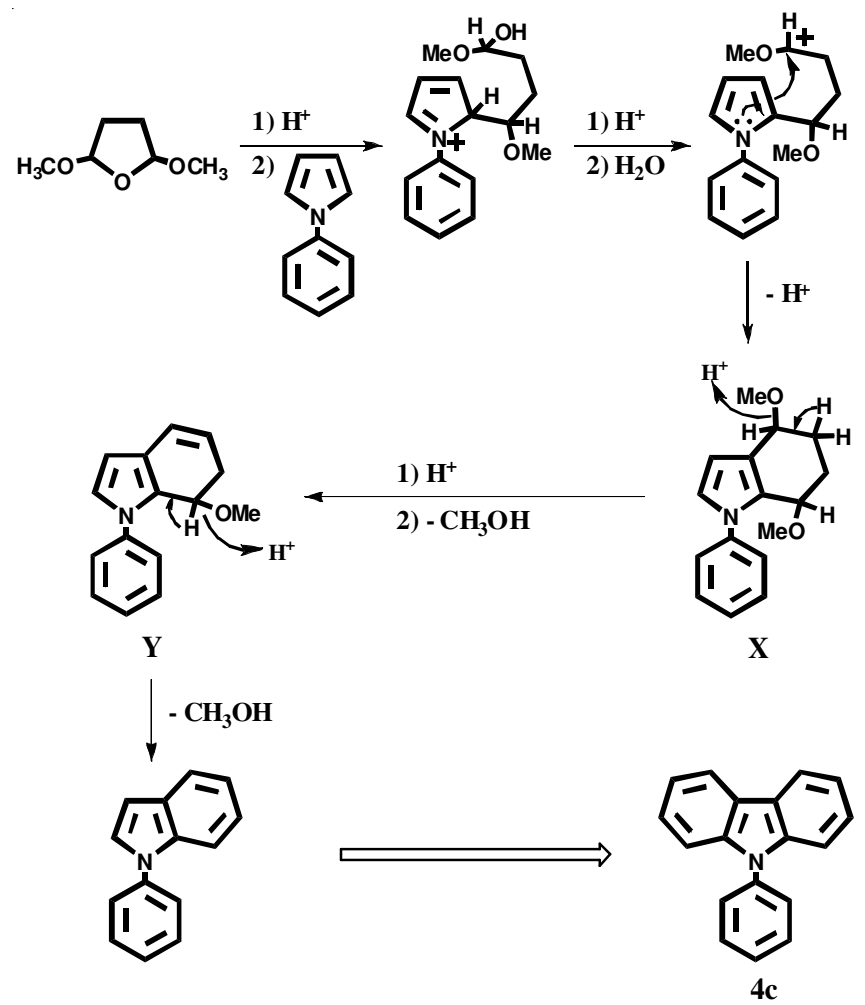

Scheme-IV: Proposed mechanism for the formation of 9-phenylcarbazole 4c 


\section{ACKNOWLEDGEMENTS}

This work was supported by the grant from Basic Science Research Institute of Dong-A University (2011).

\section{REFERENCES}

1. (a) B. Robinson, The Fischer Indole Synthesis; John Wiley and Sons: Chichester, (1982); (b) G.W. Gribble, J. Chem. Soc. Perkin Trans., 1045 (2000) and references cited therein; (c) G.R. Humphrey and J.T. Kucthe, Chem. Rer., 106, 2875 (2006); (d) B. Török, M. Abid and G. London, K.S. Angew. Chem., Int. Ed., 44, 3086 (2005).

2. (a) M. Somei and F. Yamada, Nat. Prod. Rep., 21, 278 (2004); (b) A Furstner, H. Szillat, B. Gabor and R. Mynott, J. Am. Chem. Soc., 120, 8305 (1998); (c) M. Somei and F. Yamada, Nat. Prod. Rep., 22, 73 (2005); (d) M. Török, M. Abid, S.C. Mhadgut and B. Török, Biochemistry, 45, 5377 (2006); (e) M. Abid and B. Török, Tetrahedron: Asymm., 16, 1547 (2005).

3. (a) M.L. Hodges, M.L. Spera, M.W. Moody and W.D. Harman, J. Am. Chem. Soc., 118, 7117 (1996); (b) A.R. Katritzky, C.N. Fali and J. Li, J. Org. Chem., 62, 4148 (1997).
4. (a) S.K. Bur and A. Padwa, Chem. Rev., 104, 2401 (2004); (b) T. Shimada, I. Nakamura and Y. Yamamoto, J. Am. Chem. Soc., 126, 10546 (2004); (c) Y. Ni-Shibayashi, M. Yoshikawa, Y. Inada, M.D. Milton, M. Hidai and S. Uemura, Angew. Chem. Int. Ed., 42, 2681 (2003); (d) D.J. Gorin, N.R. Davis and F.D. Toste, J. Am. Chem. Soc., 127, 11260 (2005); (e) O.V. Larionov and A. de Meijere, Angew. Chem. Int. Ed., 44, 5664 (2005); (f) Y. -Q. Fang and M. Lautens, Org. Lett., 7, 3549 (2005).

5. C. Kashima, S. Hibi, T. Marugama and T. Omote, Tetrahedron Lett., 27, 2131 (1986).

6. D.I. Jung, Y.Y. Kim, B.G. Yoo, Y.G. Lee and S.K. Choi, J. Korean Chem. Soc., 37, 982 (1993).

7. D.I. Jung, Y.Y. Kim, B.G. Yoo, Y.G. Lee and S.K. Choi, Bull. Korean Chem. Soc., 15, 168 (2004).

8. N. Elming and N. Clauson-Kass, Acta. Chem. Scand., 6, 867 (1952).

9. Y. Chiang, R.L. Hinman, S. Teodoropulos and F.B. Wipple, Tetrahedron, 23, 745 (1967).

10. A.D. Josey, Org. Syn. Coll., 5, 716 (1973).

11. A. Mohammed, T. Liliana and T. Béla, Tetrahedron Lett., 48, 4047 (2007). 